\title{
Das sagt die Industrie
}

W eniger ist oft mehr - dies gilt auch bei Praxisneueinrichtungen - Modernisierungen oder Umbauten. Je weniger externe Geräte in einen Behandlungsablauf eingebunden werden müssen, desto effizienter und ergonomischer kann gearbeitet werden. Diesbezüglich sollte bei der Praxiseinrichtung grundsätzlich Behandlungseinheiten der Vorzug gegeben werden, die eine platz- und kostenspa- rende Integration bzw. Adaptation verschiedener Systeme/Geräte erlauben, bei gleichzeitig deutlich ergonomischen Vorteilen für den Behandler. Eine zukunftssichere Einrichtung muss Flexibilität für spätere Anpassungen an veränderte Rahmenbedingungen bieten. Ein prospektiv erweiterbares Einrichtungskonzept hilft Folgekosten massiv in Grenzen zu halten. Offene Systeme, die nachträglich an unterschiedliche Arbeitsweisen und
Behandler angepasst werden können, bieten hier wertvolle Vorteile.

Darüber hinaus reduziert eine sinnvolle Anordnung der Möbel um die Dentaleinheit herum Fahr- und Greifwege während der Behandlung auf ein absolut notwendiges Minimum.

Jan Kucher // Vertriebsleiter Dentale Einrichtungen und CAD/CAM KaVo Dental GmbH, Biberach

\section{Rückengerecht: die bewegte Praxis}

Um Rückenschmerzen zu vermeiden, empfehlen Ergonomieexperten „bewegtes Sitzen“. Das belastet die Wirbelsäule und Muskulatur weniger und versorgt die Bandscheiben besser. Ideal sind ein dynamischer Wechsel zwischen Sitzen, Stehen und Gehen sowie aktives, bewegtes Sitzen und wechselnde Sitzpositionen über den Arbeitstag. Aktivsitzmöbel haben diverse individuelle Anpassungsmöglichkeiten wie Sitzfläche, Rückenlehne, Anlehndruck und Sitztiefenfederung oder Beckenkammstütze. Stehpulte und Steh-Sitz-Schreibtische ergänzen das Mobiliar. Auch PC-Tastaturen und Computermäuse sollten ergonomisch sein.

\section{Innenraumbegrünung ist Gesundheitsmanagement}

Ein schlechtes Raumklima führt zu Kopfschmerzen und macht müde. Im Winter, wenn die relative Luftfeuchtigkeit auf weniger als 30 Prozent absinkt, reizt trockene Luft die Schleimhäute. „Mit Pflanzen gestaltete Büroräume werden als erfrischender, abwechslungsreicher und konzentrationsfördernder erlebt“, haben Forscher der Universität Würzburg festgestellt. „Grünpflanzen wirken als natürliche Schalldämmer und Luftbefeuchter." Zudem verbrauchen sie Kohlenstoffdioxid und liefern Sauerstoff. Die Echte Aloe kann beispielsweise 90 Prozent
Formaldehyd abbauen, Efeu 90 Prozent Benzol und die Grünlilie mehr als 96 Prozent Kohlenstoffdioxid. Die Grünlilie verdunstet ebenso wie die Birkenfeige oder die Strahlenaralie viel Wasser. Bei Betrachtung grüner Pflanzen sinken Blutdruck und Pulsfrequenz. Es wird Stress abgebaut und die Atmung ruhiger. Kein Wunder, dass Innenraumbegrünung in vielen Unternehmen zum Gesundheitsmanagement gehört.

\section{Fazit}

Was will ich? Wer sind meine Patienten? Was brauche ich, und wie kann mein Team optimal arbeiten? In der Einrichtung der eigenen Zahnarztpraxis drücken sich die Persönlichkeit des Inhabers und die Praxisphilosophie aus. Weil die Übernahme oder Neugründung viel Geld kostet, sollte ausreichend Zeit in die Planung investiert werden. Inspirationen für durchdachte und bewährte Konzepte bieten Fachpublikationen oder Veröffentlichungen von Dentaldepots und Praxiseinrichtern. Auch wenn nicht jeder Zahnarzt seine Gestaltungsgeheimnisse gern in der Öffentlichkeit preisgibt, lohnt sich eine Anfrage in einer Praxis, die einem gefällt. Dabei sind jedoch nicht nur Farben und Designdetails spannend, sondern vor allem funktionale Besonderheiten.

\section{Hier steht eine Anzeige.}

